\title{
Gold Beam Losses at the AGS Booster Injection*
}

\author{
S.Y. Zhang and L.A. Ahrens \\ AGS Department, Brookhaven National Laboratory, Upton, NY, USA
}

\begin{abstract}
Gold beam injection efficiency decreases in proportional to the beam loss in the AGS Booster. A close look shows that large number of electrons, ions, and neutral particles are created when the gold beam scrapes wall. To investigate the neutral particle production due to the beam loss, local vacuum measurement was made during the 1998 run. It shows that the pressure created by the Booster $\mathrm{Au}^{31+}$ beam loss at injection has a $35 \mathrm{~ms}$ decay time constant. The beam loss created pressure bump in the ring is about 20 meters long. When $3 \times 10^{9}$ Gold ions scrapes wall, a pressure higher than $10^{-7}$ Torr was created. The beam lifetime calculated using these parameters is in agreement with the observed one.
\end{abstract}

\section{Introduction}

In [1], it is reported that the Gold beam Booster injection efficiency decreases in proportional to the beam loss in the ring. It is also observed that the beam life time decreases after a beam scraping in the ring. These results suggest that the gold ion lost in the ring has negative impact on the beam survival.

A close look at the effects of lost gold ions shows that there are large numbers of electrons, neutral particles, and ions (mostly positive) created when the gold beam scrapes wall $[2,3]$. It was found that the secondary electrons and ions are not the major culprit of the beam loss.

To investigate the neutral particle production due to the beam loss, vacuum measurement was performed during the 1998 run. The findings are as follows.

1. Vacuum pressure created by the Booster $\mathrm{Au}^{31+}$ beam loss at injection has a $35 \mathrm{~ms}$ decay time constant.

2. Crashing $3 \times 10^{9}$ gold ions into the wall, a pressure higher than $10^{-7}$ Torr was observed, up from the normal $5 \times 10^{-11}$ Torr.

\footnotetext{
* Work performed under the auspices of the US Dept. of Energy
}

3. The beam loss created vacuum bump in the ring is about 20 meters long.

4. The beam lifetime calculated using these results is in agreement with the observed one, i.e. about 20 ms for high intensity injection.

5. A rough estimate shows that at Booster injection, a scraping $\mathrm{Au}^{31+}$ ion could produce as much as $10^{5}$ molecules.

\section{Vacuum Pressure due to Beam Loss}

The usual vacuum measurement, through the ion gauge controller, reports mean values over several seconds. This is because that the signal obtained from ion gauge is in $p A$ level for a vacuum pressure at a few units of $10^{-11}$ Torr, whereas the noise can easily reach a $n A$ level. To investigate the beam loss created vacuum pressure structure, therefore, the ion gauge controller has to be bypassed.

Using an electrometer, Keithley 480 Picoammeter, ion gauges at different locations in the Booster ring were directly measured for the vacuum pressure due to the lost gold beam.

The time constant of Keithley 480 has a dependence on the source capacitance. For example, at the $n A$ range, a source capacitance 230 pf implies a time constant of $250 \mathrm{~ms}$. It is found that this time constant applies to most ion gauges we used in the measurement. The dynamic model of the electrometer was obtained by the calibration.

\section{Time structure of the pressure}

The time structure of the vacuum pressure created by the beam loss was studied as follows.

1. The beam was crashed in the ring, and the response of the electrometer for the ion gauge was obtained.

2. Set up a vacuum pressure with a peak value and a decay time constant. The response of electrometer is obtained by a simulation using the calibrated first order delay model of electrometer.

3. Match the simulated vacuum pressure time structure to the observed response of the 
electrometer, by adjusting the peak value and the time constant.
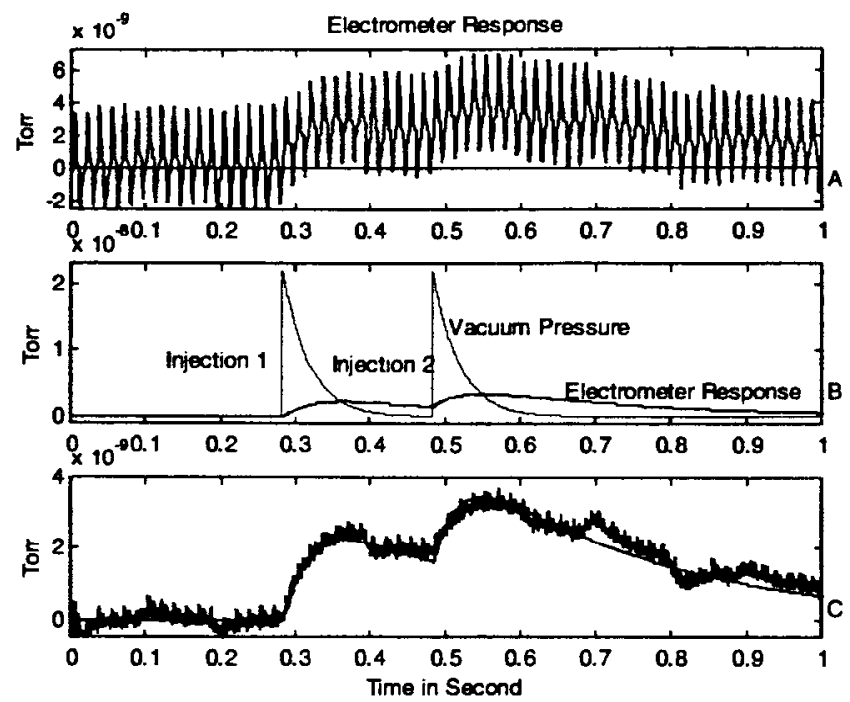

Fig.1

One example of the observed response of the electrometer is shown in Fig.1A. The location is C3B, immediate down stream of the injection septum. The injected beam scraping on the septum wall was controlled by the horizontal steering magnet immediate up stream the septum. Two Booster injections occurred in $200 \mathrm{~ms}$, and $5 \times 10^{8}$ ions were estimated lost. The vacuum pressure reported through the ion gauge controller was $5 \times 10^{-10}$ Torr.

In Fig.1B, vacuum pressure with peak of $2.2 \times 10^{-8}$ Torr and a decay time constant of $35 \mathrm{~ms}$ is shown, together with the simulated response of electrometer.

In Fig.1C, the measured response of electrometer, smoothed by a $60 \mathrm{~Hz}$ notch filter, and the simulated one are shown to be matched. It is found, therefore, that the beam loss created pressure has a decay time constant of $35 \mathrm{~ms}$.

Six experiments performed in different locations in the ring, with different intensities, have showed consistent results of a $35 \mathrm{~ms}$ decay time constant. The losses were from $3 \times 10^{8}$ ions to $10^{9}$ ions in these cases.

With larger beam losses, and higher vacuum pressure, the observed vacuum decay time was a little longer. The example shown in Fig.2 represents about $15 \times 10^{9}$ beam loss. The vacuum pressure reported through the ion gauge controller was $15 \times 10^{-9}$ Torr.

Under the condition of very high pressure, the functioning of the ion pumps is likely to be different, also the nonlinearity of the ion gauge may be involved.
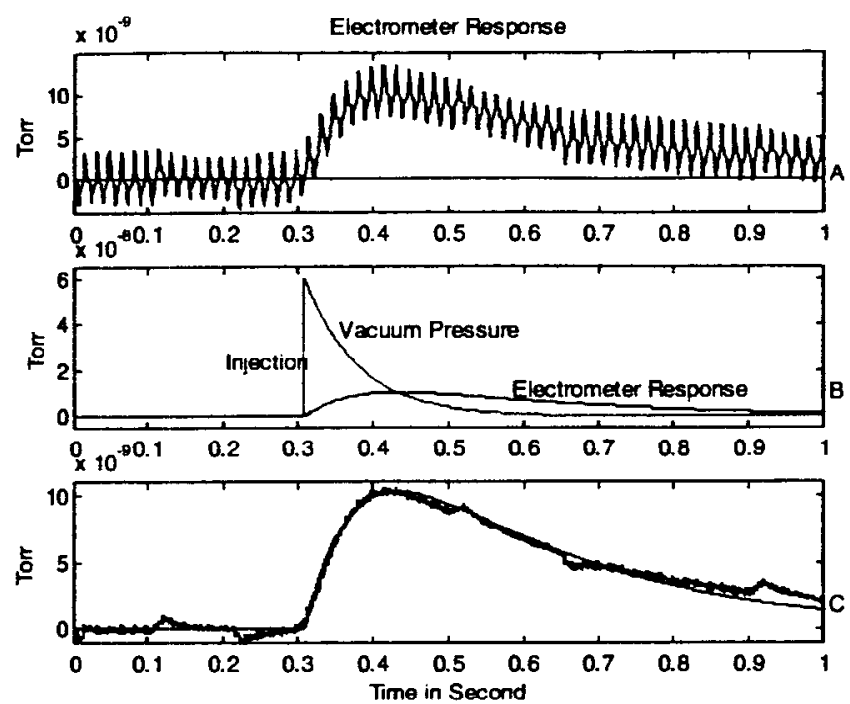

Fig.2

\section{Peak pressure}

The Booster vacuum pressure reported through the ion gauge controller is the average value over the whole cycle, 3 seconds. Taking the vacuum pressure decay time of $35 \mathrm{~ms}$, this pressure accounts for only about $1 \%$ of the real pressure created by the beam loss.

By crashing about $3 \times 10^{9}$ ions into the Booster injection inflector, a pressure higher than $2 \times 10^{-9}$ Torr was observed through the ion gauge controller. This implies that the real vacuum pressure is higher than $10^{-7}$ Torr.

\section{Pressure bump in the ring}

To observe the vacuum pressure bump in the ring, the gold beam was crashed into several locations. The vacuum pressure recorded is simply the one observed in the control room, through the ion gauge controller. One example is shown in Table 1.

\begin{tabular}{l|lllllll}
$\begin{array}{l}\text { Ion } \\
\text { Gange }\end{array}$ & C6 & C7 & C8 & D1 & D2 & D3 & Unit \\
\hline Distance & 0 & 4.2 & 8.4 & 12.6 & 16.8 & 21 & m \\
Normal & 0.3 & 0.2 & 0.1 & 0.0 & 0.2 & 0.2 & $\begin{array}{c}10^{-10} \\
\text { Tor }\end{array}$ \\
Scraping & 0.8 & 1.4 & 2.0 & 4.5 & 1.0 & 0.6 & $\begin{array}{l}10^{-10} \\
\text { Torr }\end{array}$
\end{tabular}

Table I

In average, the vacuum bump created by the beam scraping is about 15 to 25 meters long. 


\section{Beam Loss Mechanism}

Electron impact capture and ionization cross sections reported in [4] for gold ions are shown in Fig.3.

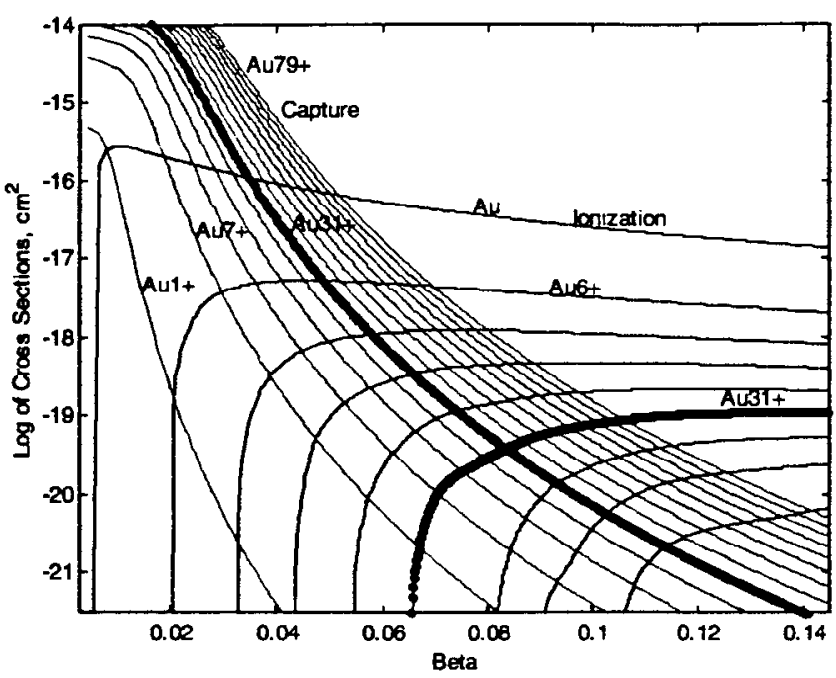

Fig.3. Electron impact cross sections. Bold lines are for $\mathrm{Au}^{31+}$

At the Booster injection energy, with $\beta=0.044$, the ionization would not occur, whereas the electron impact capture cross section is about $10^{-17} \mathrm{~cm}^{2}$ for $A u^{31+}$ ions.

The sputtering can create neutral particles, in forms of particles, molecules, or clusters. Also, the high energy secondary electrons could stimulate gas desorption, which also generate neutral particle. For convenience, we simply consider the product of the lost ions as molecules.

Taking the molecules created by the lost gold beam as $\mathrm{N}_{2}$ equivalent, the capture cross section can be taken as $\sigma_{C} \approx 10^{-16} \mathrm{~cm}^{2}$ for $A u^{31+}$ ions.

Assuming that at high intensity run, the vacuum bump created by the beam loss is 20 meters long, with the pressure of $P_{p}=10^{-7}$ Torr, the molecule density can be calculated by,

$$
n_{m}=3.3 \times 10^{16} P_{p}
$$

The beam life time is estimated by,

$$
\tau_{\text {beam }}=\frac{1}{\sigma_{C} n_{m} \beta c} \times \frac{201}{20}
$$

where $c$ is the speed of light, and 201 meter is the Booster circumference. We get $\imath_{\text {beam }} \approx 23 \mathrm{~ms}$.
The beam lifetime calculated using these parameters is in agreement with the observed one. In Fig.4, The gold beam current in about $10 \mathrm{~ms}$ starting from the injection of a typical high intensity run is shown. The Booster injection intensity was $5.2 \times 10^{9}$ ions, whereas $2 \mathrm{~ms}$ after the stacking, the beam intensity was $2.4 \times 10^{9}$ ions. About $2.8 \times 10^{9}$ gold ions were lost in less than $3 \mathrm{~ms}$. The beam life time was about $18 \mathrm{~ms}$, from $1 \mathrm{~ms}$ to $5 \mathrm{~ms}$ after the stacking.
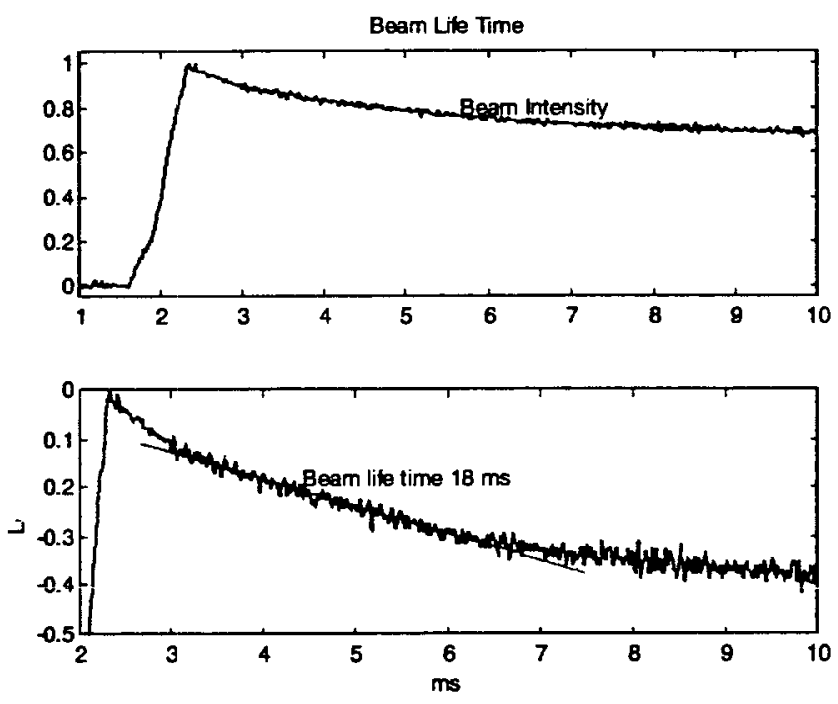

Fig.4

\section{Molecules Created by the Lost Ions}

The molecules created by one lost gold ion can be calculated by,

$$
N_{\text {mulec. }}=3.3 \times 10^{16} P_{p} V_{\ell} / N_{\text {loss }}
$$

The resulted molecules per lost ion is in the order of $10^{5}$.

\section{Acknowledgment}

We like to thank H.C. Hseuh, M. Mapes, T. Roser and $P$. Thieberger for valuable discussions, we also like to thank V. Usack and D. Warburton for technical assistant in the study.

\section{Reference}

1. S.Y. Zhang and L.A. Ahrens, EPAC98, p.2149, 1998.

2. Sputtering by Particle Bombardment III, Edited by R. Behrisch and K. Wittmaack, Springer-Verlag, N.Y., 1991.

3. S.Y. Zhang, AGS Tech Note, No.477, May, 1998.

4. S.Y. Zhang, AGS Tech Note, No.482, Dec., 1998. 